\title{
A Contextual Concept of Science
}

\author{
Sumei Cheng \\ Institute of Philosophy, \\ Shanghai Academy of Social Sciences, \\ 1610 West Zhongshan Road Xuhui District, \\ Shanghai 200020, China \\ E-mail:csm@sass.org.cn
}

\begin{abstract}
The contextual concept of science regards a scientific theory as a whole simulation for the mechanism of natural world under the given context. It argues that a scientific theory is to understand the reality only in the sense of intentionality in the process of reality simulation, rather than to describe reality in the sense of one-to-one correspondence. This concept of understanding reality is totally different from that of describing reality. Compared with the realist approaches and the anti-realist approach, the contextual approach has the following advantages: (1) it contributes to bridge the communication between the preachers of scientism and the humanists; (2) it has helped to solve the problem of underdetermination faced by scientific realism; (3) it is relatively easy to understand the correction about the concept and theory; (4) it could reflect the true process of science more properly. Therefore, it is a more promising and convincing new perspective to understand science.
\end{abstract}

Keywords: concept of science, contextualism, metaphor, realism

\section{Introduction}

How to understand the relationship between scientific theory and the world is a central topic in contemporary philosophy of science. However, there are many views in the history of philosophy of science. Realists, represented by Wilfrid S. Sellars, John J. C. Smart, Hilary Putnam and others, understand science as description of the world or take it for granted that scientific theory is correct at least in its essentials. Even where science turns out not to be strictly true, they 
are still apt to consider science as the best exemplification of rationality and cognitive progress. By contrast, anti-realists, represented by Paul Feyerabend, Larry Laudan, Bas C. van Fraassen, sociologists of scientific knowledge and so on, strongly want to overthrow the traditional scientific view based on the correspondence theory of truth. They hold that the scientific theory is a useful tool for the explanation of phenomena, or the results of social construction, and so on. These views have threatened greatly the status of objectivity which science should keep. Although these criticisms against the traditional concept of science have aroused wide academic influence, their concepts of science are no better, no more secure, objective, or rationally grounded than realists' view.

Besides, the image of a traditional concept of science, which was described by such properties as certainty, objectivity, inerrability and universality, to some extent, has been rejected by the naissance of non-Euclidean geometry and quantum mechanics. It requires us to rethink the problems of objectivity of science based on the development of contemporary sciences and the dimension of society. According to the results of scientific research in the contemporary time, if we consider the scientific theory as talking about world rather than describing the world, the models of theory as a simulation for the world, the objectivity and truthfulness of science as a human's cognitive degree for the world, rather than the relation property about correspondence between objectivity and subjectivity, then we will not need to worry about taking the opposite extreme once the contextual property and inerrability of scientific knowledge is found. And we will not need to defend the objectivity of science only by means of rejecting the culture of humanity. This is a new concept of science which can be called a "contextual concept of science".

In this paper, firstly, I will examine the traditional concepts of science and its crisis faced. Secondly, I will rely on the current achievements of sciences and the constructive process of a scientific theory and rethink the relationship between scientific theory and the world, rather than the comparative analysis between the images of science of realists and anti-realists, to explore the roots of the conflict between scientific images of scientific realist and anti-realist. In this process, I will construct a new contextual concept of science which not only accepts the reasonable critiques from relativists but also keeps the status of objectivity that science was born with. Finally I will reveal the main advantages of this contextual approach and draw a conclusion. 


\section{The traditional concept of science and its crisis}

Science was known as an embodiment of truth and a rational project in the traditional history of science. It seems that scientific laws are universal and effective, and scientific terms have their denotation. The picture of the world described by theories is true and believable. Scientific theory is the result of discovery, rather than invention, and the scientific discourse is describing the world. I call this concept of science since Renaissance a traditional concept of science. It did not need to be defended before the early years of the twentieth century. The origin of this view is from the natural sciences based on Newtonian mechanics and then it becomes a conventional understanding of science. Some philosophers of science, such as Putnam, argued that mature or well-developed scientific theories, such as physical sciences, have described the way the world really is. Others argued that those scientific theories are highly verisimilar. Bas van Fraassen (1980, p. 8) described it as, "Science aims to give us in its theories a literally true story of what world is like, and acceptance of a scientific theory involves the belief that it is true. This is the correct statement of scientific realism."

According to Marcello Pera (1994), this traditional image of science is made up of two components, which must be examined separately although traditionally they are interconnected: the epistemic and the methodological. The traditional scientific epistemology has long believed that science is based on the given evidences. Scientists get the knowledge about reality in terms of evidences. Once the knowledge is acquired, it will become a truth and be accepted. Pera argued that there are two ways to acquire knowledge.

They can be experimental, like Galileo's 'sensory experience', or intellectual, like Descartes's 'clear and distinct ideas'. In both cases, they guarantee that scientific knowledge grasps reality, either because reality reveals itself through a process starting with pure perception or because the structure of reality manifests itself through a chain of inferences stemming from the pure principles of the mind (Pera, 1994, p. 2),

for example, dynamic laws in the classical mechanics, the basic postulates in Euclidean geometry. The uses of the scientific methods ensure that we can get evidences rationally and that scientific understanding can grasp the reality, get truth and stop the controversies in these two ways. This point of view is consistent with Wittgenstein's early image of science. 
Even though the naissance of non-Euclidean geometry and its application, and the physical revolution about theory of relativity and quantum mechanics, have made some scientists think that scientific law is effective only in a given domain, rather than universally, which has brought about the new epistemological revolution and challenged the traditional philosophical categories and doubted the reliability of scientific methods, the traditional image of science did not give rise to the suspicion of logical empiricists, represented by Rudolf Carnap, and sociologists of science, represented by Robert K. Merton. On the one hand, logical empiricists rejected simply the relationship between scientific theory and the world as the problem of metaphysics; on the other hand, they exposed the logical relation between sentences and between theory and observation based on the inerrability of empirical facts. Merton was a little doubtful of the reliability and universality of scientific knowledge and considered scientists as a special group. He tried to provide the social mechanisms to ensure that scientists get the true knowledge through the sociological analysis of scientific community.

However, since Kuhn expounded the incommensurability between paradigms of theory, the new ideas, in which the observation is theory-laden and the fact is value-laden, have reached a consensus among most of philosophers of science since the 1960s. The thesis of underdetermination, especially, has challenged the realists' traditional image of science. Relativists would have us believe that scientific theories have no special claim to validity and veracity and that there is no neutral evidence to make a choice between two rival theories. More accurately, the sociologists of scientific knowledge indicated that there are inevitably many social factors in the process of scientific inquiry. Anthropologists stressed that scientific knowledge is not universal but conditional. Humanists exposed that power has taken an important role in the process of scientific knowledge production and that we ourselves are also products of a scientific culture, thus we cannot step outside the presuppositions of scientific culture to compare the legitimacy of its claims with nonscientific culture. As Andrew Ross (1991, p. 11) has said, this concept of science greatly eroded the authority of traditional image of science, which believes that scientific knowledge is context-free.

Those debates between realism and relativism about how to understand the nature of scientific knowledge have become a new issue among philosophers since the 1970s. As Larry Laudan (1997) concluded, where the realist sees the history of science as a triumphal march ever closer to the truth, the epistemic relativist sees nothing more than a succession of rival and mutually incompatible representations, each reflecting various subjective and transitory interests. 
"Where the realist sees progress in the history of science, the relativist sees only change. The realist believes that science comes as close to truth and objectivity as is humanly possible; the relativist fears that he is probably right!" (Laudan, 1997, p. 139) We can note that the concepts of science between realists and relativists are mutually exhaustive rivals. Whereas realist sees the success of science as a sign of the objectivity of scientific theory, relativist sees the success of science as context-dependent.

These debates bring about many fundamental problems related to "understanding science", which deserve much retrospection. Realists are required to explain again such concepts as objectivity, rationality, truth, the progress of science, and rethink the role of evidence and observation on the process of selection of theory, and reflect or reevaluate the limitation of traditional concept of science. They should consider the interaction among ethical, practical and cognitive factors, and study the relationship between the logical structure of science and cognitive ability of agents, and further analyse the role of the image, model, and metaphor of mature sciences.

According to the traditional image of science, political and social factors are the potential "contaminated source" of good science during scientific activity. They should be excluded. However, researchers in the field of cultural and social studies of science argued that the limitation of scientific methodology and the sociality of scientific activity in terms of scientific practice stressed various kinds of artificial factors in the form of knowledge. They believe if the increase of scientific knowledge and the judgment of science are not explained only by the evidences, logic and scientific methods, social, historical and political factors will go into the explanation of the knowledge. Hence in the process of production of knowledge, social conditions and political factors will no longer become "the contaminated source" of knowledge to be eliminated. They will be the premises or fundamental elements of production, preservation, extension and change of knowledge. In this sense, those social factors function as a dimension and an influence, together with the factors of evidence and rationality, and have assumed a reasonable place in the production of knowledge and the selection of theory, consequently, they finally make the traditional concept of science slip into the crisis. 


\section{Theory and reality in context}

Epistemic realism, or to be more precise, 'scientific realism' based on Newtonian mechanics, has become an orthodoxy among philosophers of science for almost a generation. Its rival, anti-realism, such as epistemic or cognitive relativism, instrumentalism, constructivism and so on, has raised various objections. However, after the debates went on for some time, both sides have realized that their own standpoints are the extremist views and are looking for a new way to understand the objectivity of science since the end of the last century. This indicates that if we give up the traditional concept of science it does not necessarily mean that the anti-realists' concept of science will be adequate. It only requires us to rethink the foundation of science and expatiate a new standpoint. I argue that these two opposite concepts of science, in fact, come from the same way of yes-or-no logical thinking, that is, the subjective factors must be eliminated for the sake of the stress on the truth and objectivity of knowledge. By contrast, the truth and objectivity of knowledge are abandoned for the sake of the stress on all kinds of non-evidence factors included in the process of the form of knowledge and the choices of theories. The latter concluded that science is a purely social construction and science cannot provide true knowledge. The former was based on the classical sciences and highlighted the successful applications of scientific achievements. The latter were based on contemporary sciences and highlighted the process of controversy in scientific activities. Both sides assumed the premises of correspondent theory of truth and believed in scientific methodology with the objectivity of scientific theories as the starting point of scientific inquiry. It is based on these potentially same premises that the criticism of cultural and social studies of science about the traditional image will inevitably go from one extreme to another. Although these new concepts of science have played an active role in revealing the limitation of the traditional concept of science, their views, the same as traditional concept of science, also cannot be universally accepted.

Their main disadvantage is that they have considered the objectivity of scientific research as the very beginning of thinking, rather than regarded the production of science research as a necessary result obtained in a process. This beginning does not leave room for the existence of subjectivity, in other words, the agents are merely witnesses of the beginning of scientific cognition. When the field of scientific inquiry goes into a submicroscopic world, which is impossible to perceive directly, the functions of other factors outside of experiential evidences become obvious in the process of scientific inquiry. If we still obey the traditional 
way of thinking, once the process of scientific inquiry is discovered to have the tendency of deviating from this beginning, then the beginning of understanding science will go in the direction of subjectivity. This is why the justification for the traditional image of sciences is liable to be in trouble and any non-traditional images of sciences easily give a stark denial to the objectivity of science. Moreover, from this beginning we are accustomed to understanding the scientific language as description of the world, the scientific theory as a collection of propositions, the correspondence between proposition and reality as the judgmental standard of truth, based on the common sense. We even regard the image described by theory as a true picture about the natural world.

However, when we rely on the productions of contemporary science, especially submicroscopic physics, and accept the reasonable criticism of those nontraditional concepts of sciences and rethink the beginning of scientific inquiry, we have discovered that the beginning of scientific inquiry in the dimension of scientific cognition actually starts with the unity between subjectivity and objectivity, rather than pure objectivity. In the subatomic level process of scientific cognition, whose objects are away from the system of human senses, when the media of scientific cognition included the measurement tools, the logic inference and so on, become the premise that makes scientific cognition possible, the objectivity and truthfulness of scientific knowledge becomes a longterm goal of scientific pursuit and will be a degree concept of human cognition, rather than the relation property about correspondence between subjectivity and objectivity. For example, quantum mechanics makes us more deeply understand the natural world than Newtonian mechanics does. But this does not mean that the Newtonian mechanics is wrong completely, but rather restricts the validation of it to macroscopy. The correctness of it depends on the scope of its application. Quantum entanglement, by contrast, is a typical feature of subatomic objects and will disappear in the macroscopy. If we accept this point of view, we will not need to worry about taking blindly the opposite extreme once the contextual property and inerrability of scientific knowledge is found and we will not need to defend the objectivity of science only by means of rejecting the subjectivity. This is a new "contextual concept of science" in epistemology. The reason why it is called "contextual" is that scientific cognition always takes place in the process of unity between subjectivity and objectivity and gradually tends to be perfect in the development of the de-contextualized and re-contextualized (Cheng $\&$ Guo, 2007, pp. 73-79). 
In this way, when we recognize that the beginning of scientific research includes the subjective factors and regard the objectivity and truthfulness of science as a goal of scientific pursuit, it is not possible for us to go to the opposite of denying objectivity of science solely due to the criticism of the traditional concept of science. We should admit that there are subjective factors from the very beginning of the process of scientific research. This view may include all standpoints of non-traditional images of sciences and keep them as various perspectives or views applied in understanding scientific process. This view does not mean that scientific research is seen as subjective fudge. The subjectivity included in the process of scientific research is always controlled partly by the information of reality and is based on the revelation and explanation for the experimental phenomena and the solution to scientific puzzles as objectively as possible.

The change of this way of thinking and the stress on the media of cognition imply a view that scientific theories have been understood as a whole simulation for the mechanism of the natural world under a given context. In the simulation activity, scientific discourses are an expression of cognitive content and an intelligent tool for the extension of cognitive domain and the introduction of a new symbol, or an expression of how to form a new point of view which embodies the ability of prediction, rather than talking about the natural world itself, that is, stating about the natural world (Majer, 1998, p. 230). Hence, the production of scientific cognition is no longer an absolute truth, independent of cognitive context. Here what is related to the world is no longer the statement, principle, and law of theories but its picture and prediction. In other words, the content of theory is only a part of its pictures, rather than the natural world itself. This view introduces a new theory of picture, which is called "the view of picture metaphor for scientific theories". In fact, it emphasizes on Heinrich Hertz's picture-conception of theories, rather than a new creation (Majer, 1998, p. 226)

Generally, three stages may be distinguished in the process of theorization about phenomena: first of all, it is the pursuit of empirical adequacy; the second stage gives explanatory theories; the last one provides interpretive theories.

In the first stage, the pursuit of empirical adequacy usually appears only when the old models of theory cannot explain the new phenomena. Here scientists infer the regularity of the change of phenomena in virtue of the relationship between experience and ideas which are constructed intuitionally. However, the regularity is only an empirical expression or a formulation. It cannot give a mechanically reasonable explanation or causal explanation for why the phenomena take place, 
for example, the introduction of quantum postulate. As we know, the discovery of new physical phenomena, such as X-ray, radioactivity, electron and the Zeeman effect, made the application of classical mechanics, which were applied to many fields successfully more than 300 years, face a serious crisis at the end of the 19th century. In order to solve the conflict between theory and phenomena, Planck, who was a German physicist, introduced the quantum postulate in terms of speculation when he devised his formula which fitted the data based on the research results on October 24, 1900. This postulate not only challenged the idea that there is no jumping in nature which was generally accepted by scientists for a long time, but it also became the turning point of the development of future physics.

In the second stage, what the explanatory theory provides is a new theoretical frame which goes beyond old theories. This theory, starting from given preconditions, systematically devised new mathematical formulae and suppositions with theoretical terms, and gave them clear physical meaning to make them part of the theory system. For example, the establishment of quantum mechanics system in the 1920s, when theoretical terms like probability wave, light-quantum, operator, etc. were invented, made the quantum postulate as a result of a natural inference, and provided generally efficient language tools for contemplating the submicroscopic physical phenomena, and thus contributed to the revolution of information technology and the growth of new science. Because of the explanation it provides, the explanatory theory is usually the one which could be tested empirically, that is to say, it could be confirmed or falsified empirically. If the prediction of an explanatory theory could be confirmed empirically, to some extent, it tells us what the world looks like. The explanatory theory provides the collection of mathematical and physical models of reality. For example, the general relativity is further confirmed by the scientists' declaration of their detection for gravity wave successfully on February 11, 2016. With a strong determination, scientists may understand the evolution of the cosmos according to general relativity.

In the third stage, the interpretative theory, different from the explanatory one, could not be confirmed empirically, nor be falsified. What it provides is metaphysical views and fundamental assumptions of reality. These assumptions, usually explanations of philosophy involved in preconditions of given explanatory theory, are based on the generalization of cognition results of the past, yet are not equal to them. "Assumptions are not intended to provide explanations but rather to interpret the world, that is, to ascribe to it or to specific domains of 
it a certain structure in terms of a certain fundamental ontology" (Pera, 1994, pp. 114). The assumptions provided by the interpretative theory can be divided into two classes. One is the general assumption for scientific inquiry, that is, the assumption suitable for any discipline. For example, nature is understandable, regular, uniform, and so on. The other is the special assumption inferred from nature, in other words, the assumption associated with the development of certain disciplines, such as deterministic causality, simplicity and no-jumping in nature, mechanism in physics, vitalism in biology and the gradualist theory of evolution in geology, etc. The relationship between explanatory and interpretative theories is asymmetric, or, these two theories do not change correspondently with each other. The change of interpretative theory will necessarily cause changes in explanatory theory; however, the reverse of it is not in the same case.

These three stages of theorization are restrictive of each other rather than linear. It decides that scientific theories understand the reality only in the sense of intentionality in the process of the simulation reality, rather than describe reality in the sense of one-to-one correspondence. The concept of understanding reality is totally different from that of describing reality. If a theory is thought of as a description for reality, as the realists have done, it may be regarded as the assertion about independent reality and utterance about the changes of behavior of the world, that is, it tells the true story about what the world is itself. The true or false of description is judged by reference to reality, that is, the true or false of theoretical proposition is judged by action of reality. To great extent, the cognitive agents play a role of "God's eyes". They can not only reveal the law of the world, but also record what they see. Consequently, the empirical facts have been assigned the advantageous statuses of inerrability, and scientific theories have been understood as a collection of laws or rules of truth. In contrast, if a theory is thought of as an understanding for reality, it may be only regarded as the expression of the cognitive content under given conditions and the indirect simulation of the mechanism of reality, rather than talking about reality directly. Indirect simulation is only a simulation of the full mechanism involving the hypothesis of metaphysics, rather than a mirror picture of one-toone correspondence. This process of simulation will be finished in the dynamic process of de-contextualization and re-contextualization. The cognitive agent, in this process, has a varied identity from a spectator and recorder to participator and constructor. This contextual approach to understand science will not only insist on the objectivity of science defended by realists, but also includes all the factors revealed by anti-realists. 


\section{The main advantages of the contextual concept of science}

This contextual concept of science results from the understanding for the formation and development of scientific theory, rather than the application and elaboration of the contemporary philosophers' contextualism. ${ }^{1}$ In particular it is different from Helen Longino's view of contextual empiricism. Longino (2002) argued that the objectivity of scientific knowledge is intersubjectivity or the result of discourse interaction among scientists. My view of contextual concept of science comes from the contemporary development of scientific theory rather than the analysis for the views of sociologists of knowledge. It is a view of modelism. This view has provided a new set of ways of thinking. It has changed not only the understanding of the concept of truth, but also, which is much more important, our understanding of the objects that scientific discourse talks about. The main differences between the traditional concept of science and the contextual concept could be summarized as follows:

\begin{tabular}{|c|c|c|}
\hline & Traditional concept of science & Contextual concept of science \\
\hline $\begin{array}{l}\text { Understanding } \\
\text { of the truth }\end{array}$ & $\begin{array}{l}\text { Insisting on the correspondence } \\
\text { theory of truth }\end{array}$ & $\begin{array}{l}\text { Taking truth for a long-term } \\
\text { goal of the scientific pursuit }\end{array}$ \\
\hline $\begin{array}{l}\text { Understanding } \\
\text { of the scientific } \\
\text { discourse and } \\
\text { terminology }\end{array}$ & $\begin{array}{l}\text { - Scientific discourse is the } \\
\text { description of the world } \\
\text { - Scientific terminology is } \\
\text { denotative in the world }\end{array}$ & $\begin{array}{l}\text { - Scientific discourse is the } \\
\text { description of the result of } \\
\text { scientific cognition and talking } \\
\text { about the world } \\
\text { - Scientific terminology is } \\
\text { denotative in the model of } \\
\text { theory, not the world }\end{array}$ \\
\hline $\begin{array}{l}\text { Understanding } \\
\text { of the scientific } \\
\text { method }\end{array}$ & $\begin{array}{l}\text { Scientific method is the tool } \\
\text { ensuring the objectivity of } \\
\text { science }\end{array}$ & $\begin{array}{l}\text { Scientific method is one of the } \\
\text { ways to acquire the scientific } \\
\text { cognition }\end{array}$ \\
\hline
\end{tabular}

1 For a more detailed discussion about various versions of contextualism in philosophy and sociology of science, see Cheng, 2011, pp. 53-60. 


\begin{tabular}{|c|c|c|}
\hline $\begin{array}{l}\text { Understanding } \\
\text { of the usage } \\
\text { of scientific } \\
\text { instrument }\end{array}$ & $\begin{array}{l}\text { Scientific instrument is only the } \\
\text { extension of sense organ }\end{array}$ & $\begin{array}{l}\text { Scientific instrument is one } \\
\text { of the media of scientific } \\
\text { cognition, which not only } \\
\text { provides subject with } \\
\text { information, but also takes } \\
\text { part in the preparation of the } \\
\text { object as the condition }\end{array}$ \\
\hline $\begin{array}{l}\text { Status of the } \\
\text { empirical fact }\end{array}$ & $\begin{array}{l}\text { Empirical fact, which is } \\
\text { inerrable, is the precondition } \\
\text { for developing scientific } \\
\text { theories and the tool for ending } \\
\text { scientific controversies, etc. }\end{array}$ & $\begin{array}{l}\text { Empirical fact, relying on the } \\
\text { context, is one of, not the only, } \\
\text { preconditions for developing } \\
\text { scientific theories, and the } \\
\text { main factors for ending } \\
\text { scientific controversies }\end{array}$ \\
\hline $\begin{array}{l}\text { Understanding } \\
\text { of the scientific } \\
\text { community }\end{array}$ & $\begin{array}{l}\text { Scientific community is a special } \\
\text { social group, characterized } \\
\text { by the spirit described by } \\
\text { Merton as: to adhere to the } \\
\text { universality, selflessness of the } \\
\text { scientific result, to advocate the } \\
\text { rationalism, and skepticism }\end{array}$ & $\begin{array}{l}\text { Scientific community is a } \\
\text { complex social group which } \\
\text { obeys the scientific norms, } \\
\text { and their value is required to } \\
\text { adjust continuously between } \\
\text { the factual judgment and the } \\
\text { value judgment (including the } \\
\text { ethics) and reach consensus in } \\
\text { controversies among multi- } \\
\text { groups }\end{array}$ \\
\hline $\begin{array}{l}\text { Understanding } \\
\text { of the } \\
\text { relationship } \\
\text { between theory } \\
\text { and reality }\end{array}$ & $\begin{array}{l}\text { The law in theory is the } \\
\text { discovery of the law of nature }\end{array}$ & $\begin{array}{l}\text { The law in theory relates to } \\
\text { the model of theory, while the } \\
\text { model of theory or the image is } \\
\text { the simulation of reality }\end{array}$ \\
\hline $\begin{array}{l}\text { Understanding } \\
\text { of the scientific } \\
\text { progress }\end{array}$ & $\begin{array}{l}\text { The scientific progress is the } \\
\text { continuous accumulation of the } \\
\text { true propositions, or the true } \\
\text { knowledge }\end{array}$ & $\begin{array}{l}\text { The scientific progress is } \\
\text { the increase of the similarity } \\
\text { between theory and reality }\end{array}$ \\
\hline
\end{tabular}

From the above we know that compared with other contemporary concepts of science, the contextual concept of science has at least the following important advantages as: 
Firstly, the contextual concept of science contributes significantly internally to bridge the communication between the preachers of scientism and the humanists. The preachers of scientism and the humanists have two extreme views to the understanding of science. The preachers of scientism, starting from the result of scientific inquiry, paid much attention to the successful application of scientific products, and magnified the objectivity and authority of science excessively; while the humanists, starting from the process of scientific inquiry, paid much attention to all artificial factors involved in the process of scientific research, and magnified the subjectivity and constructive properties of science excessively. It is a natural thing that they have started from the same precondition and supposition, but come to opposite conclusions and positions due to different perspectives and focuses. So if we accept the lessons of contemporary science, we could probably provide these two opposite views with a communicating platform to make both sides realize that it is equally biased to reduce social science to physics like the logical empiricists or to reduce physics to social science like the feminists. Actually, the process of scientific cognition is a gradual process of understanding the world. To the extent of regarding theory as the description of reality, van Fraassen took himself for an anti-realist. However, to another extent of regarding theory as talking about reality, he should fall into the group of realists.

Secondly, to the extent of epistemology, it is relatively easy to understand, from the contextual concept of science, why the theory, though proven to be false afterwards, has had a positive effect on research context of that day, which cannot be answered from the view of traditional philosophy. The contextual concept of science has helped to solve the problem of underdetermination faced by scientific realism, thus, it has provided good defense for the supposition supported by scientific realists that theory always converges to the truth. It also powerfully criticizes the suspicions faced by scientific realism, and even helps to dispel concerns about the incommensurability among theories. In the history of science, theories that are later proven to be false are not meaningless. However, even true theories are confined within their suitable areas, and consequently make the social constructivism of science and all non-traditional perspectives become different stages in the evolution of theory. Take mathematics as an example. For a long time it was believed that Euclidean geometry, because of the self-evidence of its precondition, was the norm providing 'pure' knowledge. Kant believed that our spatial perception definitely corresponded with Euclidean geometry because Euclidean geometry was one of our intuitive forms. In other words, our experience always corresponded with Euclidean geometry. However, after the 
birth of non-Euclidean geometry systems which were based on the innovative work of Bernhard Riemann and Hermann Minkowski, the advantageous position of Euclidean geometry was totally turned around. Moreover, scientists discovered that our universal geometry was not always what was described in Euclidean geometry.

Thirdly, to the extent of methodology, it is relatively easy to understand the correction about the concept and theory. The more abstract and complex the scientific research is, the more apparent and direct the subjective factors in the research will be, the more important the communication and cooperation among scientists will be, and the more distinct the contextual characteristics of scientific inquiry will be. For instance, at the end of the $19^{\text {th }}$ century, the successful application of classical mechanics made all physicians believe that the edifice of theoretical physics was already built up and further development was only to increase the precision. However, when many new experiment phenomena could not be explained properly within the existing frame, the motivation for searching new theories came up. Later, the birth of relativity and quantum mechanics not only provided a refreshed explication of a new form of physical theory, the meaning of time, space, mass, energy, causation, determination, probability, measurement and phenomena and the relationships among them, a new concept of time-space and mass-energy, and new concepts like non-locality, statistical causation, but also introduced new lessons of epistemology and selections of methodology.

Fourthly, to the extent of axiology, the contextual concept of science could reflect the true process of science more properly. As the inevitable product of antifoundationalism, anti-essentialism, eliminating scientism, etc., the contextual concept of science has indicated that these elements of history, society, culture and psychology are essential during the scientific practice. It learns from the characteristics of methodology of scientific hermeneutic and scientific rhetoric and excels the approach to philosophy of science taken by logical empiricism. It also bridges up the communication between scientism and humanism, rationalism and irrationalism, absolutism and relativism. Therefore, this concept has become a more promising and convincing new perspective. 


\section{Conclusion}

The contextual approach to understanding science regards a scientific theory as the understanding of reality, not the description, but this does not mean that we have to give up the instinct and pursuit that science could probably make us know the reality, because the cognition of reality is always the intended purpose of scientific inquiry. Furthermore, we have no reason to give up the ontological precondition that reality exists independently, because without reality, the intended object of scientific research, let alone science, does not exist. Contrarily, this understanding indicates more obviously that the research instinct of scientists, experiment instrument, language, inference rule, etc. play very important roles when the vision of scientific research goes beyond the confinement of human senses. It is purely a matter of depending on context as to the explanation of instrument, the understanding of semantics and pragmatics of language and concept, and the flexible practice of inference rule.

On the one hand, the contextual concept of science holds that the pursuit of truth is the cause of science, and agrees on the objectivity and truthfulness, to some extent, of the reality talked by the theory. It refutes any groundless suspicion and deconstruction of scientific knowledge. On the other hand, the contextual concept of science recognizes the historical and social features embodied in scientific knowledge, and accepts the view that the defending and rhetoric factors involved in the process of scientific cognition are also an inquiry, an argument and an explanation of scientific truth. It is likely that this kind of epistemology will make a reasonable correction and perfection of the traditional epistemology which regards scientific theory as the description of reality. It is the epistemology right between the traditional and the relativist epistemology. We call it contextual epistemology.

\section{Acknowledgements}

I acknowledge, with sincerest thanks, the reviewer's opinions, who repeatedly put forward many constructive opinions for my paper. 


\section{References}

Cheng, S. (2011), 'Contextual approaches and its problem domain of philosophy of science,' Academic Monthly, vol. 43, no. 8, pp. 53-60.

Cheng, S. \& Guo, G. (2007), 'On the concept of truth of contextualism,' Philosophical Researches, no. 5, pp. 73-79.

Laudan, L. (1997), 'Explaining the success of science: beyond epistemic realism and relativism,' in A. I. Tauber (ed.) Science and the Quest for Reality: Main Trends in the Modern World, New York: New York University Press, pp. 137-161.

Longino, H. E. (2002), The Fate of Knowledge, Princeton: Princeton University Press.

Majer, U. (1998), 'Heinrich Hertz's picture-conception of theories: its elaboration by Hilbert, Weyl, and Ramsey,' in D. Baird, R. I. G. Hughes \& A. Nordmann (eds.) Heinrich Hertz: Classical Physicist, Modern Philosopher, Dordrecht: Kluwer Academic Publishers, pp. 225-242. https://doi.org/10.1007/978-94-015-8855-3_13

Pera, M. (1994), The Discourses of Science, transl. by C. Botsford, Chicago \& London: The University of Chicago Press.

Ross, A. (1991), Strange Weather: Culture, Science, and Technology in the Age of Limits, London: Verso Press.

van Fraassen, B. (1980), The Scientific Image, Oxford: Clarendon Press. https://doi.org/10.1093/0198244274.001.0001

Sumei Cheng is professor and vice-director at the Institute of Philosophy, Shanghai Academy of Social Sciences, China, and associate editor of the journal Philosophical Analysis. 